\title{
SARS-CoV-2: a potential trigger for subacute thyroiditis? Insights from a case report
}

\author{
S. Ippolito ${ }^{1}$ (I) F. Dentali ${ }^{2} \cdot$ M. L. Tanda ${ }^{1}$
}

Received: 15 May 2020 / Accepted: 26 May 2020 / Published online: 2 June 2020

(C) Italian Society of Endocrinology (SIE) 2020

Keywords De Quervain's thyroiditis · Subacute thyroiditis · COVID-19 · SARS-CoV-2 · Post-viral thyroiditis

Dear Editor,

Routine assessment of thyroid function during hospitalization for COVID-19 is not recommended by the World Health Organization clinical management guidelines [1]. However, worsening of pre-existing thyroid dysfunction or de novo occurrence of thyroid disease, possibly caused by infection itself, should not be missed, to avoid misleading work-up, unnecessary medicalization, and its potential negative prognostic impact [2].

We hereby describe a case of thyrotoxicosis occurred during hospitalization for COVID-19. A 69-year-old woman experienced mild fever, cough, and dyspnea during the recovery phase following back surgery. A naso-pharyngeal swab test for SARS-CoV-2 was positive, and chest highresolution computed tomography (without iodinated contrast agents) showed bilateral ground glass areas typical of SARS-CoV-2-related interstitial pneumonia. The patient was then hospitalized at our dedicated COVID-19 department. The patient had longstanding non-toxic nodular goiter with a dominant benign nodule in the right lobe, and repeatedly documented euthyroidism. Because of recent surgery, she was under treatment with high-dose painkillers, including tramadol, acetaminophen, and low-dose morphine in case of severe pain. Non-steroidal anti-inflammatory drugs (NSAIDs) were not prescribed because of hypersensitivity.

S. Ippolito

silviaippolito.md@gmail.com

1 Endocrine Unit, Department of Medicine and Surgery, University of Insubria-Ospedale Di Circolo Di Varese, ASST Dei Sette Laghi, Viale Borri, 57, 21100 Varese, Italy

2 Internal Medicine Unit, Department of Medicine and Surgery, University of Insubria-Ospedale Di Circolo Di Varese, ASST Dei Sette Laghi, Viale Borri, 57, 21100 Varese, Italy
Medical therapy with hydroxychloroquine plus lopinavir/ ritonavir and low-flow oxygen therapy were initiated as prescribed on hospital admission. No iodine-containing drugs were given. From day 5, the patient started complaining of palpitations, insomnia, and agitation, despite being afebrile and clinically stable. She had no neck pain. Thyroid function assessment showed suppressed serum thyroid-stimulating hormone (TSH: $0.08 \mathrm{mU} / \mathrm{l}$, normal range $0.27-4.2$ ) with increased serum-free thyroxine (FT4: $24.6 \mathrm{pg} / \mathrm{ml}$, normal range 0.3-17) and free triiodothyronine (FT3: $5.5 \mathrm{pg} / \mathrm{ml}$, normal range 2-4.4). TSH-receptor antibodies, thyroperoxidase, and thyroglobulin antibodies were all negative.

Empirical therapy with methimazole was initiated. Five days later, thyrotoxicosis worsened (TSH $0.02 \mathrm{mU} / \mathrm{l}, \mathrm{FT} 4$ $29.7 \mathrm{pg} / \mathrm{ml}$, FT3 $5.6 \mathrm{pg} / \mathrm{ml}$ ), and serum thyroglobulin was elevated (187 $\mu \mathrm{g} / \mathrm{l}$, normal range 3.5-77). Bedside thyroid ultrasound showed an enlarged hypoechoic thyroid, decreased vascularity and the known 30-mm homogeneous nodule in the right lobe (with peripheral vascularization). At thyroid scan using Tc 99-m, there was no uptake. Because NSAIDS could not be employed, methimazole was discontinued and steroids were given, starting with $40 \mathrm{mg}$ intravenous methylprednisolone for 3 days, then continuing with $25 \mathrm{mg}$ oral prednisone, to be progressively tapered over 4 weeks or more, according to clinical response [3]. Within a few days, symptoms markedly improved; 10 days after starting steroids, biochemical thyrotoxicosis substantially improved (FT4 $21.9 \mathrm{pg} / \mathrm{ml}$; FT3, $3.07 \mathrm{pg} / \mathrm{ml}$ ). Of note, naso-pharyngeal control swab test for SARS-CoV-2 resulted positive 2 months after the first diagnosis, though respiratory symptoms were completely solved.

Clinical presentation, ultrasound features, lack of thyroidal uptake, high serum thyroglobulin levels, and the absence of thyroid autoantibodies suggest a thyroid-destructive process compatible with a diagnosis of subacute $(D e$ Quervain's) thyroiditis, possibly triggered by SARS-CoV-2 
infection. Neck pain was absent, but the patient was under high doses of painkillers after back surgery, possibly masking local symptoms. At differential diagnosis, we could not exclude painless thyroiditis, which, however, is less likely for epidemiological reasons and for the lack of thyroid autoimmunity.

This and another case report [4] of destructive thyroiditis associated with SARS-CoV-2 infection were almost simultaneously described. Of note, other viruses involved in subacute thyroiditis were found in thyroid tissues, and autopsies conducted after SARS outbreak in 2002-2003 found extensive damage of follicular thyroid epithelium, which represents the histopathological counterpart of destructive thyroiditis [5]. Indeed, a potential localization of SARS-CoV-2 in thyroid tissue would clarify the persistent viral positivity, long after resolution of respiratory manifestations. Despite the strong clinical suspicion of an association between SARS-CoV-2 and subacute thyroiditis in this patient, this likely hypothesis cannot be definitively proven.

In conclusion, we reported a case of subacute (destructive) thyroiditis during hospitalization for COVID-19, potentially related to SARS-CoV-2 infection, effectively treated by steroids. Physicians working in COVID-19 departments should be aware of possible connections between SARSCoV-2 and thyroid dysfunction, which should be investigated by prospective studies.

Acknowledgements The authors want to thank colleagues working in COVID-19 department [Flavio Tangianu MD, Benedetta Pennella MD, Alessandra Grossi MD, Silvia Lepanto MD and Bohdan Patera $\mathrm{MD}$ ] and the other endocrinological consultants [Adriana Lai MD, Elvira Masiello MD] for the cooperation in evaluating and managing the patient and for their comments and suggestions on the manuscript.

Funding The authors received no specific funding for this work.

\section{Compliance with ethical standards}

Conflict of interest All authors declare that they have no conflict of interest.

Ethical approval All procedures performed during this retrospective study were in accordance with the ethical standards of institutional and/ or national research committee and with the 1964 Helsinki Declaration and its later amendments or comparable ethical standards. The ethical committee approval is not required for case reports.

Informed consent Signed consent was obtained from the patient. Data were anonymized.

\section{References}

1. World Health Organization (2020) Clinical management of severe acute respiratory infection (SARI) when COVID-19 disease is suspected: interim guidance. https://apps.who.int/iris/handle/10665 /331446 License: CC BY-NC-SA 3.0 IGO

2. Bartalena L, Chiovato L, Marcocci C, Vitti P, Piantanida E, Tanda ML (2020) Management of Graves' hyperthyroidism in time of COVID-19 pandemic. J Endocrinol Invest. https://doi. org/10.1007/s40618-020-01293-7

3. Ross DS, Burch HB, Cooper DS et al (2016) 2016 American Thyroid Association guidelines for diagnosis and management of hyperthyroidism and other causes of thyrotoxicosis. Thyroid 26(10):1343-1421. https://doi.org/10.1089/thy.2016.0229

4. Brancatella A, Ricci D, Viola N, Sgrò D, Santini F, Latrofa F (2020) Subacute Thyroiditis After Sars-COV-2 Infection. J Clin Endocr Metab 105(7):dgaa276. https://doi.org/10.1210/clinem/ dgaa276

5. Desaillud R, Hober D (2009) Virus and thyroiditis: an update. Virol J 6:5. https://doi.org/10.1186/1743-422X-6-5

Publisher's Note Springer Nature remains neutral with regard to jurisdictional claims in published maps and institutional affiliations. 